\title{
Analysis of ischaemic crisis using the informational causal entropy-complexity plane
}

Walter Legnani, Francisco Traversaro, Francisco O. Redelico, Leandro J. Cymberknop, Ricardo L. Armentano, and Osvaldo A. Rosso

Citation: Chaos 28, 075518 (2018); doi: 10.1063/1.5026422

View online: https://doi.org/10.1063/1.5026422

View Table of Contents: http://aip.scitation.org/toc/cha/28/7

Published by the American Institute of Physics

\section{Articles you may be interested in}

Bandt-Pompe symbolization dynamics for time series with tied values: A data-driven approach

Chaos: An Interdisciplinary Journal of Nonlinear Science 28, 075502 (2018); 10.1063/1.5022021

Detecting directional couplings from multivariate flows by the joint distance distribution

Chaos: An Interdisciplinary Journal of Nonlinear Science 28, 075302 (2018); 10.1063/1.5010779

An analysis of high-frequency cryptocurrencies prices dynamics using permutation-information-theory quantifiers Chaos: An Interdisciplinary Journal of Nonlinear Science 28, 075511 (2018); 10.1063/1.5027153

Causal network reconstruction from time series: From theoretical assumptions to practical estimation

Chaos: An Interdisciplinary Journal of Nonlinear Science 28, 075310 (2018); 10.1063/1.5025050

State space reconstruction of spatially extended systems and of time delayed systems from the time series of a scalar variable

Chaos: An Interdisciplinary Journal of Nonlinear Science 28, 075504 (2018); 10.1063/1.5023485

Is human atrial fibrillation stochastic or deterministic?-Insights from missing ordinal patterns and causal entropy-complexity plane analysis

Chaos: An Interdisciplinary Journal of Nonlinear Science 28, 063130 (2018); 10.1063/1.5023588

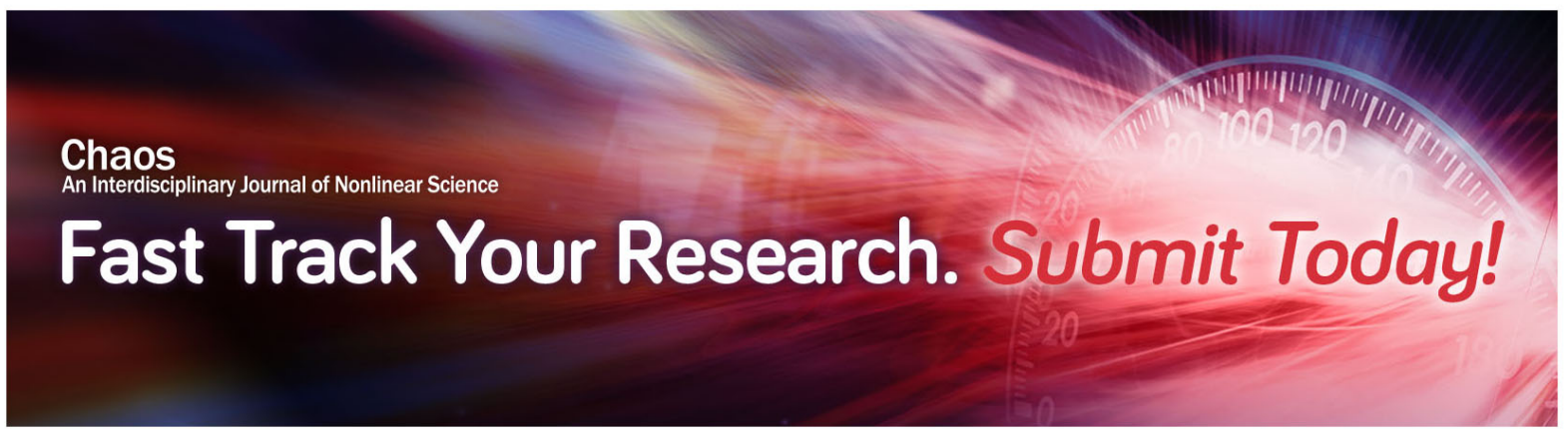




\title{
Analysis of ischaemic crisis using the informational causal entropy-complexity plane
}

\author{
Walter Legnani, ${ }^{1, a)}$ Francisco Traversaro, ${ }^{2, b)}$ Francisco O. Redelico, ${ }^{3,4, c)}$ Leandro J. Cymberknop, ${ }^{5, d)}$ \\ Ricardo L. Armentano, ${ }^{5, \mathrm{e})}$ and Osvaldo A. Rosso ${ }^{3,6, f)}$ \\ ${ }^{1}$ Signal and Image Processing Center (CEPSI), Universidad Tecnológica Nacional, Facultad Regional Buenos Aires, Medrano \\ 951, C1179AAQ Ciudad Autónoma de Buenos Aires, Argentina \\ ${ }^{2}$ Grupo de Investigación en Sistemas de Información, Universidad Nacional de Lanús \& CONICET, 29 de Septiembre 3901, \\ B1826GLC Lanús, Buenos Aires, Argentina and Instituto Tecnólgico de Buenos Aires (ITBA) \& CONICET, Av. Eduardo Madero \\ 399, C1181ACH Ciudad Autónoma de Buenos Aires, Argentina \\ ${ }^{3}$ Departamento de Informática en Salud, Hospital Italiano de Buenos Aires \& CONICET, C1199ABB Ciudad Autónoma de Buenos \\ Aires, Argentina \\ ${ }^{4}$ Departamento de Ciencia y Tecnología, Universidad Nacional de Quilmes, Roque Sáenz Peña 352, B1876BXD Bernal, Buenos \\ Aires, Argentina \\ ${ }^{5}$ Grupo de Investigación y Desarrollo en Bioingeniería (GIBIO and Signal and Image Processing Center (CEPSI), Universidad \\ Tecnológica Nacional, Facultad Regional Buenos Aires, Medrano 951, C1179AAQ Ciudad Autónoma de Buenos Aires, Argentina \\ ${ }^{6}$ Instituto de Física, Universidade Federal de Alagoas, Av. Lourival Melo Mota, s/n, 57072-970 Maceió, Alagoas, Brazil and \\ Complex Systems Group, Facultad de Ingeniería y Ciencias Aplicadas, Universidad de los Andes, Las Condes, 12455 Santiago, \\ Chile
}

(Received 19 February 2018; accepted 11 June 2018; published online 30 July 2018)

\begin{abstract}
In the present work, an ischaemic process, mainly focused on the reperfusion stage, is studied using the informational causal entropy-complexity plane. Ischaemic wall behavior under this condition was analyzed through wall thickness and ventricular pressure variations, acquired during an obstructive flow maneuver performed on left coronary arteries of surgically instrumented animals. Basically, the induction of ischaemia depends on the temporary occlusion of left circumflex coronary artery (which supplies blood to the posterior left ventricular wall) that lasts for a few seconds. Normal perfusion of the wall was then reestablished while the anterior ventricular wall remained adequately perfused during the entire maneuver. The obtained results showed that system dynamics could be effectively described by entropy-complexity loops, in both abnormally and well perfused walls. These results could contribute to making an objective indicator of the recovery heart tissues after an ischaemic process, in a way to quantify the restoration of myocardial behavior after the supply of oxygen to the ventricular wall was suppressed for a brief period. Published by AIP Publishing. https://doi.org/10. $1063 / 1.5026422$
\end{abstract}

In this work, we analyze the evolution of an ischaemic process using causal Information Theory descriptors. The obtained characterization could be used for quantitative evaluation of the restoration of myocardial behavior after the supply of oxygen to the ventricular wall was suppressed for a brief period.

\section{INTRODUCTION}

Coronary arteries supply the heart muscle with oxygenrich blood. Without an adequate blood supply, heart muscle becomes starved of oxygen and may become permanently damaged (acute myocardial infarction). Coronary blood flow, at least to the left ventricle, occurs predominantly in diastole since the resistance vessels within the heart are occluded during systolic contraction. ${ }^{1}$ Most cases of infarction are due to

\footnotetext{
a)walterlegnani@gmail.com

b) ftraversaro@itba.edu.ar

c) francisco.redelico@gmail.com

d)1jcymber@ieee.org

e) gibio@frba.utn.edu.ar

f) oarosso@gmail.com
}

the formation of an occluding thrombus superimposed on a lipid-rich atherosclerotic plaque. Impairment of coronary flow (and thus oxygen) to target tissues is known as "myocardial ischaemia." 2 Myocardial ischaemia is responsible for angina, unstable angina, and, less commonly, shortness of breath secondary to ischaemic left ventricular dysfunction (angina equivalent) as well as cardiac arrhythmias. Patients with severe coronary disease may have ischaemia to such a degree that subendocardial infarction may ensue. ${ }^{1}$ Time elapsed during an ischaemia crisis determines the severity of the clinical consequences suffered by any organ, in this case the heart, specifically the left ventricle. Then, the ischaemic process finishes when the reperfusion is initiated. If the time elapsed during an ischaemic crisis is extended, longer than $15 \mathrm{~min}$, the damage to the heart tissue is usually irreversible. Long periods of severe ischaemia (longer than $15 \mathrm{~min}$ ) followed by reperfusion produce irreversible damage that culminates in loss of viable myocardium function. ${ }^{3}$ In this sense, cardiac tissue may activate endogenous mechanisms of cell survival resulting in physiological conditions of adaptation to ischaemia, known as myocardial stunning, ischaemic preconditioning, and myocardial hibernation, which sustains cardiac cell survival in the context of oxygen deprivation and during the stress of reperfusion. ${ }^{4}$ 
In a previous study, nonlinear measures to time series arising from the cardiac ischaemic process were carried out. ${ }^{5}$ Loss of complexity of ventricular wall thickness (VWT) variations was quantified, and fractal dimension assessment (waveform structural information) was proposed as an "intrinsic marker" of the development of an ischaemic process. We found that this kind of mathematical tool has behaviour closely similar to the clinical community ischaemic indices in defining the ischaemic process. Subsequently, chaotic metrics were applied to VWT pulsatility in Ref. 6, where a non-linear analysis was carried out under baseline conditions through the phase space reconstruction, determinism evaluation, and largest Lyapunov exponent assessment of the time series. It was concluded that the nonlinear system characterization of VWT behavior could be related to a chaotic process.

The use of quantifiers based on Information Theory, like entropy and statistical complexity, evaluated using the symbolic methodology proposed by Bandt and Pompe $^{7}$ in order to estimate the required probabilities (which take into account in a natural way the time causality of generated time series) has led to interesting results regarding the characterization of nonlinear chaotic dynamics, improving the understanding of their associated time series. In particular, allowing to define a causal entropy-complexity plane a diagnostic tool for discrimination of stochastic and chaotic signals, being its application goes across several disciplines, like physics, ${ }^{8-17}$ hydrology and geophysics, ${ }^{18-22}$ random and pseudorandom numbers, ${ }^{23-25}$ biology, medicine, and econophysics, ${ }^{26-32}$ among others. Even though these measures were successfully used to distinguish between chaos and noise, in this paper we are interested in characterizing the physiological states during an ischaemic process instead of the generating nature of dynamics itself.

The present work is organized as follows: in Sec. II, the Information Theory based quantifiers are introduced. In Sec. III, a short description of signal analysis methodology corresponding to signals of interest during the ischaemic crisis is provided. Finally, the analysis of results and discussion of them, as well as conclusions, are given in Sec. IV.

\section{INFORMATION THEORY BASED QUANTIFIERS}

For a given $\mathcal{X}(t)=\left\{x_{t}: t=1, \ldots, M ; x_{t} \in \mathbb{R}\right\}$, a discrete random variable (a discrete time series provided by a physical process) with $M<\infty$ possible values and whose distribution is characterized by the discrete probability density function (PDF) $P=\left\{p_{j} \geq 0: j=1, \ldots, N ; p_{j} \in \mathbb{R}\right\}$ and $\sum_{j=1}^{N} p_{j}=1$, the logarithmic Shannon entropy ${ }^{33}$ is given by

$$
\mathrm{S}[P]=-\sum_{j=1}^{N} p_{j} \ln \left(p_{j}\right)
$$

and provides a measure of the information embedded into the physical process described by $P$, and it is a bounded function, $0 \leq \mathrm{S}[P] \leq \mathrm{S}_{\max }$, implying full certainty and full uncertainty in both limits, respectively. We define the normalized Shannon entropy by $\mathcal{H}[P]=\mathrm{S}[P] / \mathrm{S}_{\max }$. In particular, for the uniform distribution $P_{e}=\left\{p_{j}=1 / N, \forall j=1, \ldots, N\right\}$, it follows that $\mathrm{S}\left[P_{e}\right]=\mathrm{S}_{\max }=\ln N$.

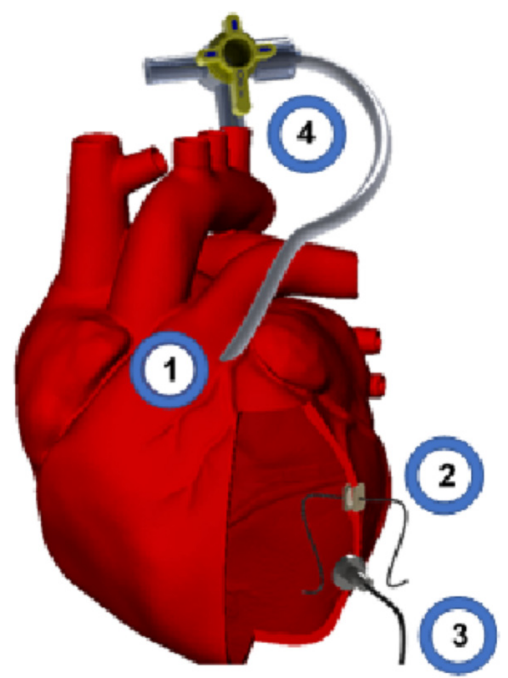

FIG. 1. Measurement scheme of left ventricular pressure (LVP) and left parietal ventricular thicknesses (LVTs). (1) Left circumflex coronary artery bypass. (2) Ultrasonic crystals for parietal thickness measurement. (3) Pressure micro transducer for LVP measurement. (4) Clamping between the subclavian and coronary arteries.

Although Shannon entropy is a good global quantifier for describing the level of order/disorder on a physical process, it does not quantify the degree of structure or patterns present in the process. ${ }^{34}$ Hence, an additional quantifier is necessary to be introduced in order to measure these hidden structures in the process in order to fully characterize the dynamical system under study; i.e., the generalized statistical complexity measure $\mathcal{C}[P]^{35,36}$ is defined as

$$
\mathrm{C}[P]=\mathcal{H}[P] \cdot \mathcal{Q}_{J S}\left[P, P_{e}\right],
$$

where $0 \leq \mathcal{H}[P] \leq 1$ is the above defined normalized Shannon entropy and the disequilibrium $0 \leq \mathcal{Q}_{J S}\left[P, P_{e}\right] \leq 1$ is defined in terms of the extensive, in thermodynamical sense, Jensen-Shannon divergence: $\mathcal{Q}_{J S}\left[P, P_{e}\right]=Q_{0} \mathcal{J}\left[P, P_{e}\right]$ and $\mathcal{J}\left[P, P_{e}\right]=\left\{\mathrm{S}\left[\frac{\left(P+P_{e}\right)}{2}\right]-\frac{1}{2} \mathrm{~S}[P]-\frac{1}{2} \mathrm{~S}\left[P_{e}\right]\right\}$, where $Q_{0}$ is a

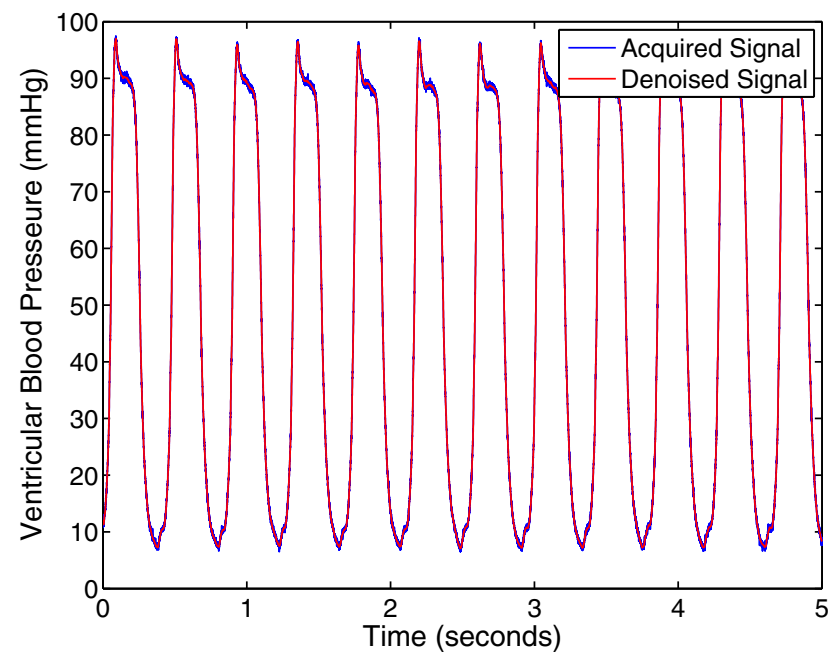

FIG. 2. Typical acquired signal (blue line) and wavelet denoised signal (red line) corresponding to $5 \mathrm{~s}$ ventricular blood pressure $(\mathrm{mm} \mathrm{Hg})$. The sample signal is $4000 \mathrm{~Hz}$. 
normalization constant given by $Q_{0}=\mathcal{J}\left[P_{0}, P_{e}\right]^{-1}$. The disequilibrium quantifies difference between two PDFs: the probability $P$ of the actual state of the system and the probability corresponding to the equilibrium $P_{e}$ (taken the uniform distribution as state of reference).

There is no a unique way to compute the probability set $P$ associated with a time series. ${ }^{37}$ The applicability and performance of each one depends on the kind of data to be analyzed, its stationarity, time series length, and noise contamination level, among other factors. Global aspects of the generating time series (TS) dynamics can be somehow captured, but the different approaches are not equivalent in their ability to discern all the relevant physical details. In particular, for the characterization and distinction between deterministic (low dimensional chaos) and stochastic dynamics, we found that "the inclusion of time causality is one of the most important features to be considered" in the methodology for PDF $P$ associated with the TS, ${ }^{38}$ a fact that can be reached using the permutation Bandt-Pompe PDF. ${ }^{7}$

In the present work, for the Bandt-Pompe PDF $(\Pi)$ associated with the time series analyzed, we follow the procedure described by Rosso and co-workers in Refs. 39 and 40. The (normalized) Shannon entropy and generalized statistical complexity, evaluated with this ordinal pattern probability distribution, are called the permutation (normalized) Shannon entropy, $\mathcal{H}[\Pi]$, and permutation statistical complexity, $\mathcal{C}[\Pi]$, respectively.

The temporal evolution of the above defined quantifiers, entropy $\mathcal{H}[\Pi]$ and complexity $\mathcal{C}[\Pi]$, can be analyzed using a two-dimensional diagram called causal entropy-complexity plane. ${ }^{38}$ The term "causality" reminds us of the fact that temporal correlations between successive samples are taken into account through BP-PDF $\Pi$, in the evaluation of Information Theory quantifiers. The second law of thermodynamics states that entropy grows monotonically with time. Then, the entropy $\mathcal{H}$ can be regarded as an arrow of time, allowing this form to follow the time evolution of a dynamical system or its changes of behavior with the different control parameter

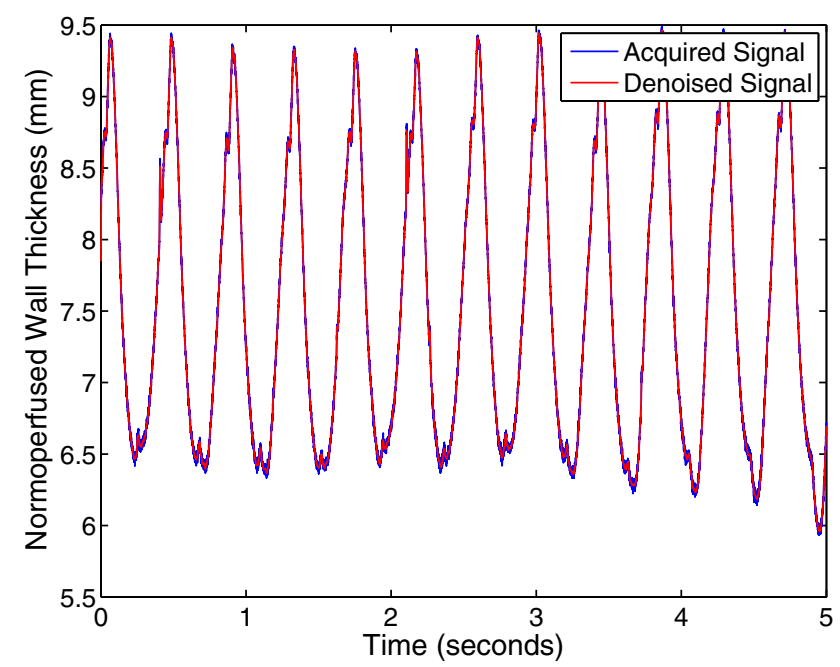

FIG. 3. Typical acquired signal (blue line) and wavelet denoised signal (red line) corresponding to $5 \mathrm{~s}$ normoperfused wall thickness $(\mathrm{mm})$. The sample signal is $4000 \mathrm{~Hz}$. values. ${ }^{37}$ In the entropy-complexity plane, all the possible values are bounded by two continuous curves which represent, respectively, the maximum $\mathcal{C}_{\max }$ and minimum $\mathcal{C}_{\text {min }}$ statistical complexity values as function of the normalized entropy $\mathcal{H}$ and can be evaluated as explained by Martín, Plastino, and Rosso. ${ }^{41}$

The causal $\mathcal{H} \times \mathcal{C}$-plane as a diagnostic tool has shown to be particularly efficient at distinguishing between the deterministic chaotic and stochastic nature of time series since the permutation quantifiers have distinctive behaviors for different types of dynamics. In particular, Rosso and co-workers ${ }^{38}$ showed that chaotic maps have intermediate entropy $\mathcal{H}$, while complexity $\mathcal{C}$ reaches larger values, close to those of the limit $\mathcal{C}_{\max }$. Moreover, similar behavior is still observed when chaotic maps' time series are contaminated with small or moderate amount of additive uncorrelated or correlated noise. ${ }^{42,43}$ For regular process, both quantifiers have small values close to zero $(\mathcal{H} \approx 0, \mathcal{C} \approx 0)$. Finally, for totally uncorrelated stochastic process, the values are close to $\mathcal{H} \approx 1$ and $\mathcal{C} \approx 0$. Correlated stochastic process (colored noises) with $f^{-k}$ power spectrum $(0<k \leq 3)$ is characterized by intermediate permutation entropy and intermediate permutation complexity values between $\mathcal{C}_{\min }$ and $\mathcal{C}_{\max }$.

It is useful to define a measure that reflects the complexity of a system given its amount of information $(\mathcal{H})$. To determine the relative position between both limit states of minimum and maximum complexity, given $\mathcal{H}$, we propose a simple measure to grasp the amount of complexity regarding the degree of information presented in the time series. We call it relative complexity $\Delta^{(\mathcal{H})}$ and define it as

$$
\Delta^{(\mathcal{H})}[\Pi]=\frac{\mathcal{C}[\Pi]-\mathcal{C}_{\min }}{\mathcal{C}_{\max }-\mathcal{C}_{\min }}
$$

Note that, for colored noises with $f^{-k}$, we obtain $\Delta^{(\mathcal{H})} \approx 0.5$ for lower and moderate $k$-values, and for the case of chaotic maps, we have $\Delta^{(\mathcal{H})} \approx 1$. By convention, in the case of $\mathcal{H}=0$ or $\mathcal{H}=1$, we take $\Delta^{(\mathcal{H})}=0$.

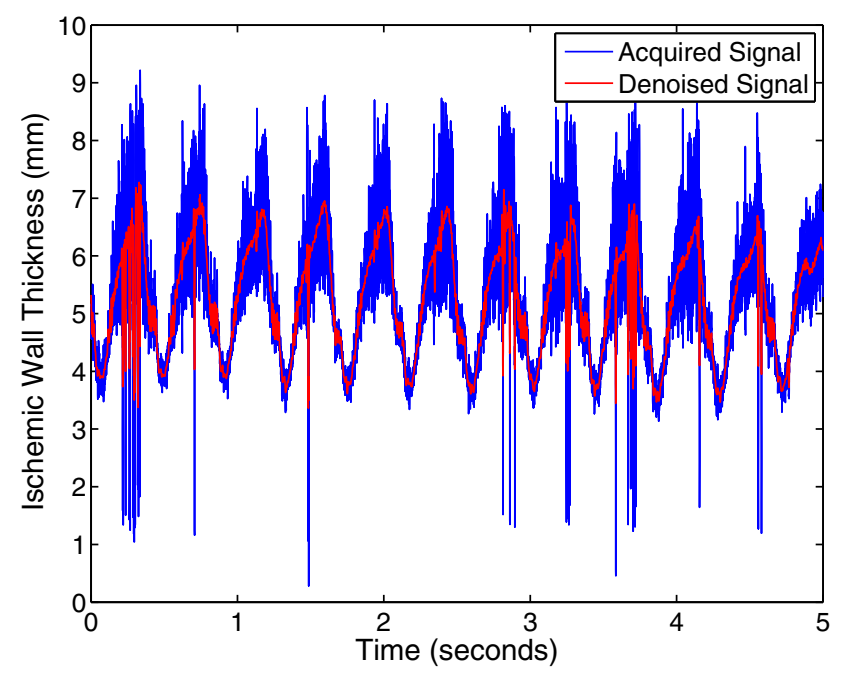

FIG. 4. Typical acquired signal (blue line) and wavelet denoised signal (red line) corresponding to $5 \mathrm{~s}$ ischaemic wall thickness $(\mathrm{mm})$. The sample signal is $4000 \mathrm{~Hz}$. 


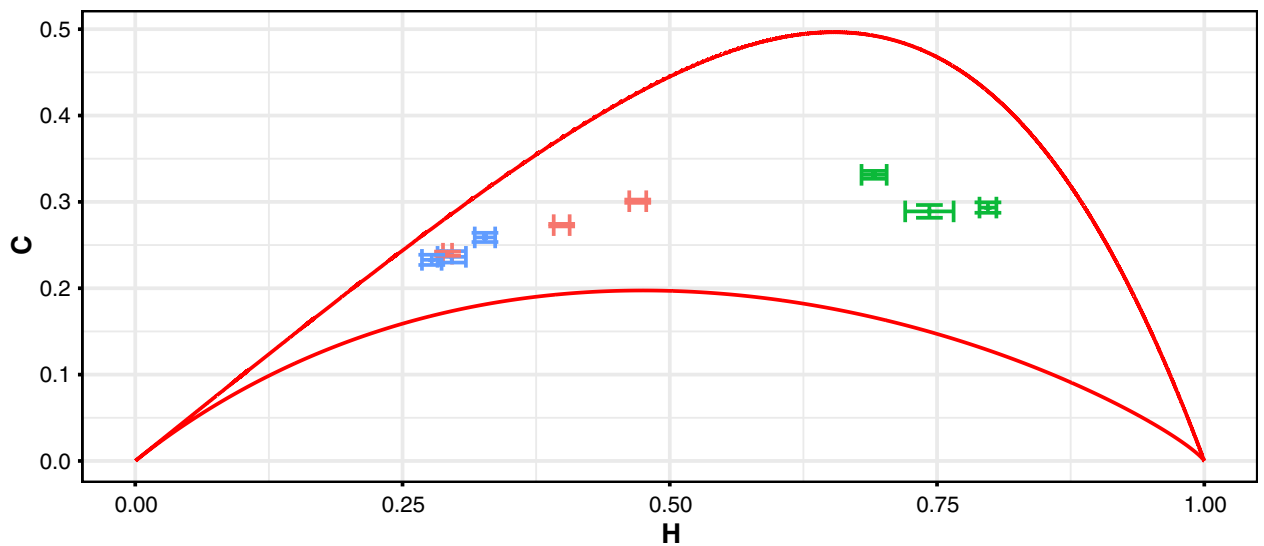

FIG. 5. Location of the left ventricular pressure (red), and both anterior (green) and posterior (blue) thickness in the $\mathcal{H} \times \mathcal{C}$ informational plane measured in the three mongrel dogs. The Bandt and Pompe PDF was calculated with $D=6$ and $\tau=16$. Mean values and standard error, for each dog, taken over time window signals of $1.5 \mathrm{~s}$ with overlap of $0.5 \mathrm{~s}$. The continuous lines correspond to $\mathcal{C}_{\max }$ and $\mathcal{C}_{\min }$ evaluated for $D=6$.

\section{MATERIALS AND METHODS}

Three mongrel dogs weighing [18.8;26.5] $\mathrm{kg}(24.4 \pm$ 3.3 , mean $\pm \mathrm{SD}$ ) were operated. We followed the surgical procedure that was originally described by Cymberknop and co-workers in Ref. 5. Figure 1 shows a scheme of a dog's heart after the surgical procedure.

For each mongrel dog, three time series (TS) were taken leading to three measures during the ischaemic procedure: the ventricular pressure (see Fig. 2), the normoperfused wall thickness (see Fig. 3), and the ischaemic wall thickness (see Fig. 4). The length of the TS varied from dog to dog, from $14 \times 10^{4}$ to $28 \times 10^{4}$ observations. The sampling frequency was set to $4000 \mathrm{~Hz}$ for every signal. In general, the biomechanical signals acquired in vivo are noisy, especially when a piezoelectric crystal is employed in the acquisition device, as in this case for the ultrasonic micro-crystals. For this reason, a pre-filtering of the signals recorded from the instrumented dogs was necessary.

The signals were preprocessed through filtering by wavelets (see Figs. 2-4 for typical examples of $5 \mathrm{~s}$ interval). Among other useful characteristics to apply in signal processing, the wavelets have the property to localize, at different scales, the features of the signal. This property is used in this work to denoise the raw signal to remove the artifacts without loosing the relevant characteristics of the information contained in the signal. To accomplish this denoising, we used the well-known procedure named wavelet denoising by thresholding. The basic idea of the method of wavelet denoising is to select the wavelet coefficient smaller than a specific level and suppress it from the wavelet transform. Assuming that they typically came from noise, the denoised signals
TABLE II. Relative complexity obtained for the three mongrel dogs.

\begin{tabular}{lccc}
\hline \hline Relative complexity $\Delta^{(\mathcal{H})}[\Pi]$ & $\operatorname{Dog} 1$ & $\operatorname{Dog} 2$ & $\operatorname{Dog} 3$ \\
\hline Ventricular pressure & 0.441414 & 0.612475 & 0.451506 \\
Normoperfused wall thickness & 0.556916 & 0.642343 & 0.585377 \\
Ischaemic wall thickness & 0.432307 & 0.550843 & 0.506375 \\
\hline \hline
\end{tabular}

could be reconstructed using the inverse wavelet transform to obtain signals with specific noise reduction, without loosing amplitude and phase shift. For details, see Refs. 44 and 45. The denoising was performed using the Daubechies order four wavelet applied with a soft thresholding method.

With each denoised signal, the normalized permutation entropy $(\mathcal{H})$ and the permutation statistical complexity $(\mathcal{C})$ were computed as stated in Sec. II using sliding windows of length 6000 data $(1.5 \mathrm{~s})$ with an overlap of 2000 data $(0.5 \mathrm{~s})$. The BP PDF requires two parameters: the pattern length $D$ and the time delay $\tau$. In accordance with the results obtained by Boardman and co-workers, in these kinds of signals, ${ }^{46}$ the best value for the time delay is $\tau=16$. All results are shown for the pattern length $D=6$ because there was no significant difference in the results when the $D$ parameter varied from $D=3$ to $D=6$.

\section{RESULTS AND DISCUSSION}

The severity and consequences of an ischaemic crisis, on any organ, especially the heart, are determined by the time elapsed and by other clinical factors concurrent with the crisis, and then the start-up of the reperfusion begins the process which finishes the ischaemic crisis. ${ }^{3,47}$ The damage to heart

TABLE I. Obtained mean values and standard error for normalized Shannon entropy and statistical complexity of the ventricular wall thickness and ventricular pressure for the three mongrel dogs. BP PDF was calculated with $D=6$ and $\tau=16$, taken over time window signals of $1.5 \mathrm{~s}$ with an overlap of $0.5 \mathrm{~s}$.

\begin{tabular}{|c|c|c|c|c|c|c|c|}
\hline & \multirow[b]{2}{*}{ Dog } & \multicolumn{2}{|c|}{ Ventricular pressure } & \multicolumn{2}{|c|}{ Normoperfused wall thickness } & \multicolumn{2}{|c|}{ Ischaemic wall thickness } \\
\hline & & Mean & SE & Mean & SE & Mean & SE \\
\hline & 1 & 0.3988766 & 0.0045199 & 0.2960285 & 0.0080353 & 0.7428525 & 0.0137216 \\
\hline \multirow[t]{3}{*}{$\mathcal{H}$} & 2 & 0.2920966 & 0.0025640 & 0.2773665 & 0.0055675 & 0.7976171 & 0.0047886 \\
\hline & 3 & 0.4700439 & 0.0048156 & 0.3271174 & 0.0057901 & 0.6911170 & 0.0071269 \\
\hline & 1 & 0.2730826 & 0.0008992 & 0.2363704 & 0.0040174 & 0.2890026 & 0.0044784 \\
\hline \multirow[t]{2}{*}{$\mathcal{C}$} & 2 & 0.2400031 & 0.0014705 & 0.2328880 & 0.0035836 & 0.2934200 & 0.0036553 \\
\hline & 3 & 0.3007864 & 0.0011493 & 0.2588568 & 0.0032338 & 0.3313376 & 0.0027667 \\
\hline
\end{tabular}



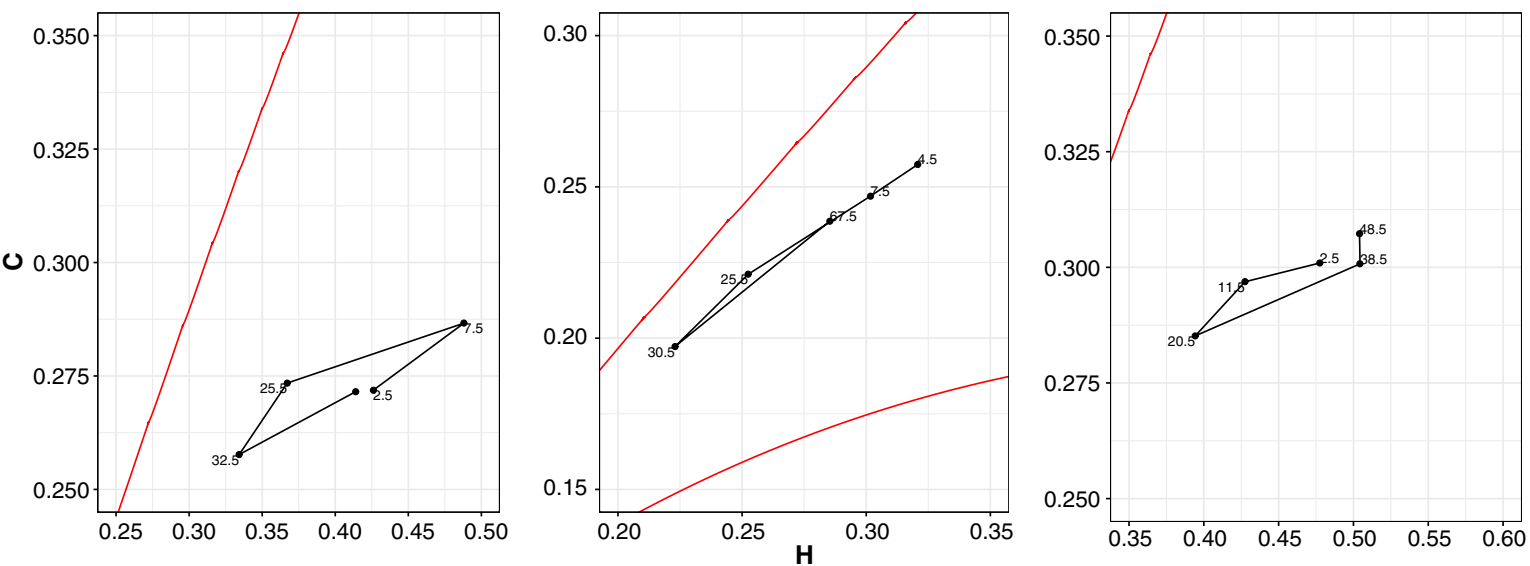

FIG. 6. Trajectory of the left ventricular pressure during the maneuver for the three different mongrel dogs in the $\mathcal{H} \times \mathcal{C}$ informational plane. The Bandt and Pompe PDF was calculated with $D=6$ and $\tau=16$, taken over time window signals of $1.5 \mathrm{~s}$ with an overlap of $0.5 \mathrm{~s}$. The numbers next to the dots are the time in seconds after the beginning of the procedure. In all the cases, the entropy and the statistical complexity decay until the wall is reperfused and return to the initial values indicating a complete recovery of basal conditions. The continuous lines correspond to $\mathcal{C}_{\max }$ and $\mathcal{C}_{\min }$ evaluated for $D=6$.

cells, cardiomyocytes, begins about 20 min after initiating the ischaemic process, and the reversibility of this kind of damage was indicated in the literature and this could take place within $60 \mathrm{~min}$ after the ischaemic process begins and reperfusion is done. ${ }^{48}$ The deep knowledge of how the damage to ischaemic process is reversible is a matter of discussion. ${ }^{3} \mathrm{~A}$ diversity of results are indicated in the literature; for example, reperfusion after a long elapsed ischaemic time process followed by an immediate reperfusion leads to strong damage to the heart, whereas cyclical periods of short ischaemic crises do a protective function of the vital organ. ${ }^{3}$

The natural dynamics of each healthy dog was altered by a surgical procedure deriving from a perturbation of those systems, which is reflected in changes in the location on the informational plane $\mathcal{H} \times \mathcal{C}$. In the ischaemic wall thickness TS, this is reflected in an augment of $\mathcal{H}$ (compared with the normoperfused wall TS) due to direct manipulation of the wall, leading to an alteration in the correlation structure of the data in which the relationship between components of the systems is underlaid (see Fig. 5 and Table I). All the measures are similar in terms of permutation statistical complexity when they are measured independent of the permutation entropy. While the pressure and thickness of the normoperfused wall are on the low entropy side, the thickness of the ischaemic wall is located on the high entropy side of the plane, and that may be due to the fact that the surgical procedure endowed the ischaemic wall with an altered dynamics.

In Table II, the relative complexity $\Delta^{(\mathcal{H})}[\Pi]$ for each measure is shown. It can be seen that there is a loss in the relative complexity [Eq. (3)] for the ischaemic wall compared with the normoperfused wall. This is consistent with the hypothesis of loss of complexity for a physiological system with age or disease. ${ }^{49-51}$

In Figs. 6-8, the trajectories on the informational plane $\mathcal{H} \times \mathcal{C}$ from the beginning of the procedure to the end of the obtained data for all three measures are shown. In these trajectories, the extension of the cycle to return to the initial conditions depends mainly on the duration of the procedure (it is worth mentioning that the scale of the figures varies on each $\operatorname{dog}$ ). In Fig. 6, the left ventricular pressure performs a cycle on the informational plane $\mathcal{H} \times \mathcal{C}$. An alteration in the time series dynamics due to the ischaemia but with a return to
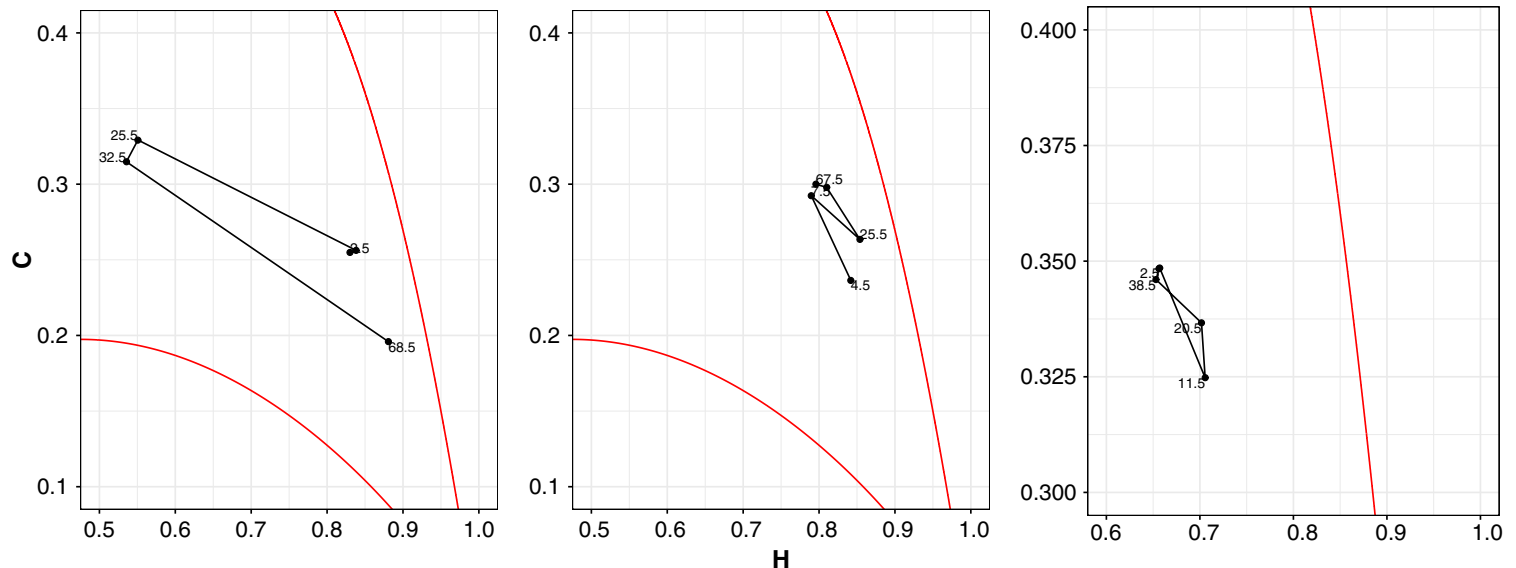

FIG. 7. Trajectory of the thickness ischaemic wall during the maneuver for the three different mongrel dogs in the $\mathcal{H} \times \mathcal{C}$ informational plane. The numbers next to the dots are the time in seconds after the beginning of the procedure. The Bandt and Pompe PDF was calculated with $D=6$ and $\tau=16$, taken over time window signals of $1.5 \mathrm{~s}$ with an overlap of $0.5 \mathrm{~s}$. This trajectory is different for each dog, indicating that the wall has been altered, agreeing with the ischaemic process. The continuous lines correspond to $\mathcal{C}_{\max }$ and $\mathcal{C}_{\min }$ evaluated for $D=6$. 

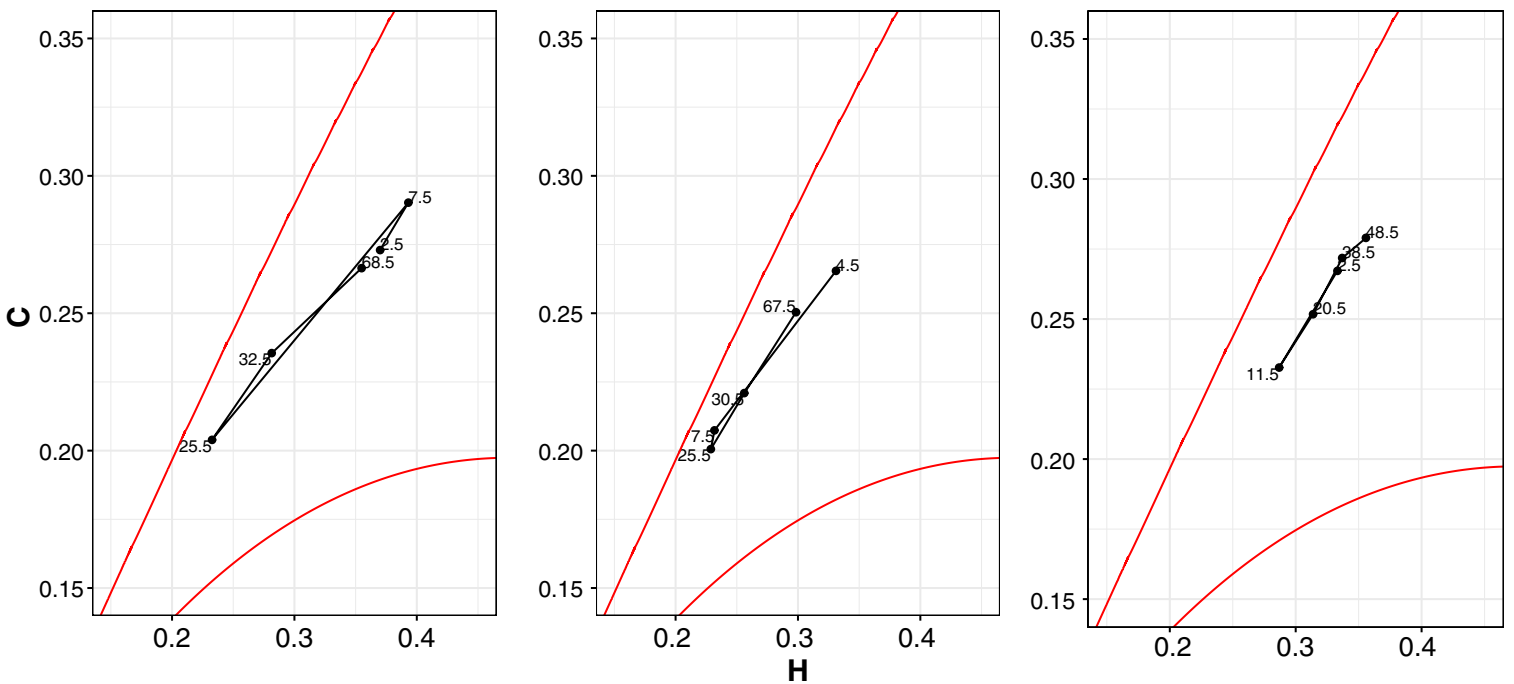

FIG. 8. Trajectory of the thickness normoperfused wall during the maneuver for the three different mongrel dogs in the $\mathcal{H} \times \mathcal{C}$ informational plane. The numbers next to the dots are the time in seconds after the beginning of the procedure. The Bandt and Pompe PDF was calculated with $m=6$ and $\tau=16$, taken over time window signals of $1.5 \mathrm{~s}$ with an overlap of $0.5 \mathrm{~s}$. In all the cases, the entropy and the statistical complexity decay until the wall is reperfused and return to the initial values indicating a complete recovery of basal conditions. The continuous lines correspond to $\mathcal{C}_{\max }$ and $\mathcal{C}_{\min }$ evaluated for $D=6$.

the initial conditions can be observed. Some type of recovery of the ventricular function may be inferred.

In Fig. 7, the ischaemic wall trajectory on the informational plane $\mathcal{H} \times \mathcal{C}$ is shown. For the first dog (Fig. 7, left), the procedure lasted the longest and could not reach the initial point in the informational plane.

In Fig. 8, a return to the initial conditions is observed and again the longest trajectory corresponds to the same dog whose procedure lasted the longest (see Fig. 8, left). The extension of the principal axis of the trajectory area (thought as an ellipse) may be used as a proxy of the duration of the ischemic crisis and could be related to the severity of the procedure on the ventricle.

In summary, we have described the evolution of the cycle Ischaemia/Reperfusion using complexity based techniques. This could have clinical implications, as the permutation entropy and statistical permutation complexity could be used as a proxy to understand the condition of the cardiac muscle. This contribution could pave the way to elaborate a methodology to construct an objective indicator of the recovery of heart tissues after an ischaemic process, in a way to illustrate the restoration of myocardial behavior after the supply of oxygen to the ventricular wall was suppressed for a brief period.

\section{ACKNOWLEDGMENTS}

To the student Martín De Luca, fellowship of the Bioengineering Group (GIBIO, FRBA, UTN), for his valuable help in enhancing the scheme of Fig. 1. This work was supported by projects UTN 2220 and UTN 4729 of the Universidad Tecnológica Nacional (Argentina).

${ }^{1}$ D. C. Crossman, Heart 90, 576 (2004).

${ }^{2}$ G. K. Hansson, New Engl. J. Med. 352, 1685 (2005).

${ }^{3}$ T. Kalogeris, C. P. Baines, M. Krenz, and R. J. Korthuis, Int. Rev. Cell. Mol. Biol. 298, 229 (2012).

${ }^{4}$ E. Rashed and C. Depre, Arch. Mal. Coeur. Vaiss. 99, 1236 (2006).

${ }^{5}$ L. J. Cymberknop, W. Legnani, J. G. Barra, F. M. Pessana, and R. L. Armentano, Physiol. Meas. 34, 83 (2012).

${ }^{6}$ W. Legnani, L. Cymberknop, M. Alfonso, F. Pessana, and R. Armentano, in VI Latin American Congress on Biomedical Engineering CLAIB
2014, Paraná, Argentina, 29-31 October 2014 (Springer, Cham, 2015), pp. $47-50$.

${ }^{7}$ C. Bandt and B. Pompe, Phys. Rev. Lett. 88, 174102 (2002).

${ }^{8}$ A. Kowalski, M. Martín, A. Plastino, and O. Rosso, Phys. D Nonlinear Phenom. 233, 21 (2007).

${ }^{9}$ O. A. Rosso and C. Masoller, Phys. Rev. E 79, 040106(R) (2009).

${ }^{10}$ L. Zunino, M. C. Soriano, I. Fischer, O. A. Rosso, and C. R. Mirasso, Phys. Rev. E 82, 0000 (2010).

${ }^{11}$ M. C. Soriano, L. Zunino, O. A. Rosso, I. Fischer, and C. R. Mirasso, IEEE J. Quantum Electron. 47, 252 (2011).

${ }^{12}$ L. D. Micco, J. G. Fernández, H. A. Larrondo, A. Plastino, and O. A. Rosso, Phys. A Stat. Mech. Appl. 391, 2564 (2012).

${ }^{13}$ L. Zunino, M. C. Soriano, and O. A. Rosso, Phys. Rev. E 86, 046210 (2012).

${ }^{14}$ A. L. Aquino, T. S. Cavalcante, E. S. Almeida, A. C. Frery, and O. A. Rosso, Eur. Phys. J. B 88 (2015).

${ }^{15}$ F. Montani, O. A. Rosso, F. S. Matias, S. L. Bressler, and C. R. Mirasso, Philos. Trans. R. Soc. A Math. Phys. Eng. Sci. 373, 20150110 (2015).

${ }^{16}$ A. L. Aquino, H. S. Ramos, A. C. Frery, L. P. Viana, T. S. Cavalcante, and O. A. Rosso, Phys. A Stat. Mech. Appl. 465, 277 (2017).

${ }^{17}$ F. O. Redelico, F. Traversaro, N. Oyarzabal, I. Vilaboa, and O. A. Rosso, Phys. A Stat. Mech. Appl. 470, 321 (2017).

${ }^{18}$ H. Lange, O. Rosso, and M. Hauhs, Eur. Phys. J. Spec. Top. 222, 535 (2013).

${ }^{19}$ P. M. Saco, L. C. Carpi, A. Figliola, E. Serrano, and O. A. Rosso, Phys. A Stat. Mech. Appl. 389, 5022 (2010).

${ }^{20}$ F. Serinaldi, L. Zunino, and O. A. Rosso, Stoch. Environ. Res. Risk. Assess. 28, 1685 (2013).

${ }^{21}$ S. Sippel, H. Lange, M. D. Mahecha, M. Hauhs, P. Bodesheim, T. Kaminski, F. Gans, and O. A. Rosso, PLoS ONE 11, e0164960 (2016).

${ }^{22}$ M. Pulido and O. A. Rosso, J. Atmos. Sci. 74, 3253 (2017).

${ }^{23}$ H. Larrondo, M. Martín, C. González, A. Plastino, and O. Rosso, Phys. Lett. A 352, 421 (2006).

${ }^{24}$ L. De Micco, C. González, H. Larrondo, M. Martín, A. Plastino, and O. Rosso, Phys. A Stat. Mech. Appl. 387, 3373 (2008).

${ }^{25}$ L. D. Micco, H. A. Larrondo, A. Plastino, and O. A. Rosso, Philos. Trans. R. Soc. A Math. Phys. Eng. Sci. 367, 3281 (2009).

${ }^{26}$ M. Zanin, L. Zunino, O. A. Rosso, and D. Papo, Entropy 14, 1553 (2012).

${ }^{27}$ A. F. Bariviera, L. Zunino, M. B. Guercio, L. B. Martinez, and O. A. Rosso, Eur. Phys. J. B 86, 509 (2013).

${ }^{28}$ F. Montani and O. Rosso, Entropy 16, 4677 (2014).

${ }^{29}$ F. Montani, E. B. Deleglise, and O. A. Rosso, Phys. A Stat. Mech. Appl. 401, 58 (2014).

${ }^{30}$ F. Montani, R. Baravalle, L. Montangie, and O. A. Rosso, Philos. Trans. R. Soc. A Math. Phys. Eng. Sci. 373, 20150109 (2015).

${ }^{31}$ A. F. Bariviera, M. B. Guercio, L. B. Martinez, and O. A. Rosso, Eur. Phys. J. B 88 (2015). 
${ }^{32}$ A. F. Bariviera, M. B. Guercio, L. B. Martinez, and O. A. Rosso, Philos. Trans. R. Soc. A Math. Phys. Eng. Sci. 373, 20150119 (2015).

${ }^{33}$ C. E. Shannon, Bell Syst. Tech. J. 27, 379 (1948).

${ }^{34}$ D. P. Feldman and J. P. Crutchfield, Phys. Lett. A 238, 244 (1998).

${ }^{35}$ P. W. Lamberti, M. T. Martín, A. Plastino, and O. A. Rosso, Phys. A Stat. Mech. Appl. 334, 119 (2004).

${ }^{36}$ R. Lopez-Ruiz, H. Mancini, and X. Calbet, Phys. Lett. A 209, 321 (1995).

${ }^{37}$ O. A. Rosso, L. De Micco, H. A. Larrondo, M. T. Martín, and A. Plastino, Int. J. Bifurcat. Chaos 20, 775 (2010).

${ }^{38}$ O. A. Rosso, H. A. Larrondo, M. T. Martin, A. Plastino, and M. A. Fuentes, Phys. Rev. Lett. 99, 154102 (2007).

${ }^{39}$ F. Traversaro, F. O. Redelico, M. R. Risk, A. C. Frery, and O. A. Rosso, Chaos Interdiscip. J. Nonlinear Sci. 28, 075502 (2018).

${ }^{40}$ O. A. Rosso, M. T. Martín, H. A. Larrondo, A. M. Kowalski, and A. Plastino, "Generalized statistical complexity: A new tool for dynamical systems," in Concepts and Recent Advances in Generalized Information Measures and Statistics, edited by A. M. Kowalski, R. D. Rossignoli, and E. M. F. Curado (Bentham Science Publishers, 2013), pp. 169-215.

${ }^{41}$ M. T. Martín, A. Plastino, and O. A. Rosso, Phys. A Stat. Mech. Appl. 369, 439 (2006)
${ }^{42}$ O. A. Rosso, L. C. Carpi, P. M. Saco, M. G. Ravetti, A. Plastino, and H. A. Larrondo, Phys. A Stat. Mech. Appl. 391, 42 (2012).

${ }^{43}$ O. A. Rosso, L. C. Carpi, P. M. Saco, M. G. Ravetti, H. A. Larrondo, and A. Plastino, Eur. Phys. J. B 85, 1 (2012).

${ }^{44}$ G. P. Nason and B. W. Silverman, Wavelets and Statistics (Springer, New York, 1995), pp. 281-299.

${ }^{45}$ R. R. Coifman and D. L. Donoho, Wavelets and Statistics (Springer, New York, 1995), pp. 125-150.

${ }^{46}$ A. Boardman, F. S. Schlindwein, and A. P. Rocha, Physiol. Meas. 23, 325419 (2002).

${ }^{47}$ Z.-Q. Zhao, M. Nakamura, N.-P. Wang, D. A. Velez, K. O. Hewan-Lowe, R. A. Guyton, and J. Vinten-Johansen, J. Surg. Res. 94, 133 (2000).

${ }^{48}$ M. Miura, H. Matsu-Oka, T. Saito, and T. Kanazawa, Jpn. Circ. J. 55, 868 (1991).

${ }^{49}$ D. Kaplan, M. Furman, S. Pincus, S. Ryan, L. Lipsitz, and A. Goldberger, Biophys. J. 59, 945 (1991).

${ }^{50}$ L. A. Lipsitz and A. L. Goldberger, J. Am. Med. Assoc. 267, 1806 (1992).

${ }^{51}$ L. J. Cymberknop, W. Legnani, F. M. Pessana, A. Crottogini, and R. L. Armentano, in 2012 Annual International Conference of the IEEE Engineering in Medicine and Biology Society (EMBC) (IEEE, 2012), pp. 4200-4203. 